\title{
The tagged photon facility at the MAX IV Laboratory
}

\author{
Bent Schroder ${ }^{1, \mathrm{a}}$ \\ ${ }^{1}$ MAX IV Laboratory, Lund, Sweden
}

\begin{abstract}
The upgraded tagged photon facility at the MAX IV Laboratory is presented. The pulsed electron beam from a linac system is stretched in the MAX I ring and the electron beam hits a thin aluminium foil in which bremsstrahlung is produced. One of two available magnetic spectrometers is used to determine the energy of the postbremsstrahlung electrons. The tagged photon ranges from 10 to $180 \mathrm{MeV}$ with an energy resolution of about $300 \mathrm{keV}$. Rates as high as $4 \times 10^{6}$ photons s${ }^{-1} \mathrm{MeV}^{-1}$ have been used. The experimental area and facilities are described as well as examples of ongoing experiments.
\end{abstract}

\section{Introduction}

The photon tagging technique is used at several laboratories as a source of quasi-monochromatic photons with known energy $\mathrm{E}_{\gamma}$ and intensity. An electron beam, with energy $\mathrm{E}_{0}$, impinges on a 50 $300 \mu \mathrm{m}$ aluminium radiator and the energy, $\mathrm{E}_{\mathrm{r}}$, of the post-bremsstrahlung electron is measured in the focal plane of a magnetic spectrometer. The photon energy is obtained from the difference $E_{0}-E_{r}$, with an energy resolution of about $300 \mathrm{keV}$. The MAX IV Laboratory is a National Facility placed in Lund, Sweden. At present the laboratory operates three electron storage rings with maximum energies of 550, 750 and $1500 \mathrm{MeV}$ used for the production of synchrotron radiation. The MAX I ring (550 $\mathrm{MeV}$ ) may also be operated as a stretcher ring converting the $100 \mathrm{~ns}$ long linac electron pulse to an almost continuous beam.

\section{The tagging facility}

The electron beam from the linac enters the magnet area, see Fig. 1, and hits the aluminium radiator placed in front of one of two magnets. The MT (Main Tagger) is used for the energy range 10 $<\mathrm{E}_{\gamma}<130 \mathrm{MeV}$ and the ET (EndPoint Tagger) covers the range $125<\mathrm{E}_{\gamma}<180 \mathrm{MeV}$. At present the maximum available electron energy is $220 \mathrm{MeV}$. The scattered electrons are detected in a 62 channel hodoscope equipped with plastic scintillators. The same hodoscope is used in the focal plane of both magnets. The non-interacting electron beam is dumped in a Faraday cup [1].

The positions of some diagnostic tools in the experimental area are shown in Fig. 1. The in beam monitor (IBM) consists of three thin scintillators (S1-S3). The coincident signals S2 and S3 are proportional to the number of photons. Scintillator S1 eliminates contributions from charged particle in the incident beam. Two beam position monitors (xy1 and xy2) are used to check the beam positions

${ }^{\mathrm{a}}$ Corresponding author: bent.schroder@nuclear.lu.se

This is an Open Access article distributed under the terms of the Creative Commons Attribution License 2.0, which permits unrestricted use, distribution, and reproduction in any medium, provided the original work is properly cited. 
before and after the target. The photon camera consists of a scintillating screen viewed by a CCD camera.

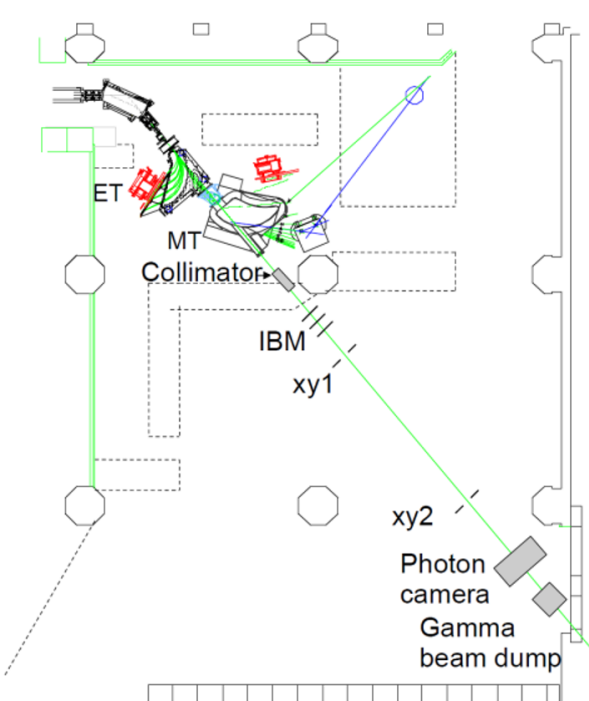

Figure 1. A 30 degree bending magnet directs the beam towards the tagging magnets, MT and ET. A simple dipole magnet is used to bring the non-interacting electron beam towards the beam dump. The diagnostic tools are described in the text.

\section{The experimental program at the tagging facility}

The experimental program at the MAX IV tagging facility contains investigations of Compton scattering on light nuclei, studies of the $\left(\gamma, \pi^{+}\right)$and $\left(\gamma, \pi^{+}\right)$reactions, measurements of the total photoabsorption cross sections in ${ }^{4} \mathrm{He}$ and Li-isotopes and detector tests for e.g. the electromagnetic calorimeter in PANDA. Experiments with linearly polarized photons are also part of the program.

\subsection{Compton scattering on ${ }^{12} \mathrm{C}$}

One of the ongoing programs at the MAX IV Laboratory is the investigation of Compton scattering on the deuteron [2] with the goal to determine the electric $\alpha_{N}$ and magnetic $\beta_{N}$ polarizabilities of the nucleon. Using the known values of the polarizabilites of the proton it is then possible to deduce $\alpha_{n}$ and $\beta_{\mathrm{n}}$, the values for the neutron [3]. The polarizabilites are structure constants related to the stiffness of the nucleons towards deformations induced by the electromagnetic fields (photons).

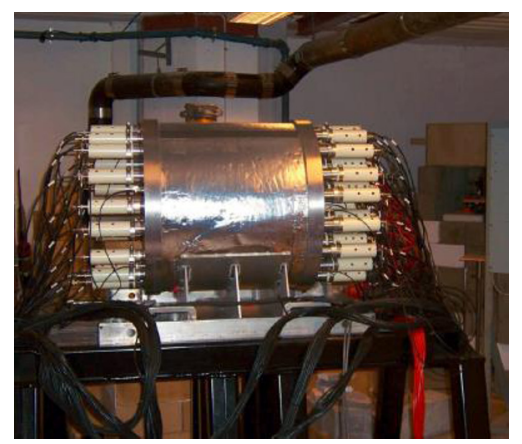

Figure 2. One of the three large $\mathrm{NaI}(\mathrm{Tl})$ crystals available for studies of the Compton scattering in deuterium and light nuclei. The dimension of all three (BUNI, CATS and DIANA) are about the same. The diameter of the central core is about $48 \mathrm{~cm}$ and the length is $50 \mathrm{~cm}$. 
Issues related to rate dependent corrections (corrections for stolen trues) have been resolved and data for the ${ }^{12} \mathrm{C}(\gamma, \gamma)$ scattering cross section at $\theta_{\gamma}=60^{\circ}$ is shown in Fig. 3. The stolen trues correction is related to the time-flight-spectrum used to identify coincident events for the photons in the $\mathrm{NaI}(\mathrm{Tl})$ detector and scattered electrons in the focal plane. A fraction of the true events, in the coincidence peak, are lost (stolen) due to random electrons. This effect increases with the electron rate.

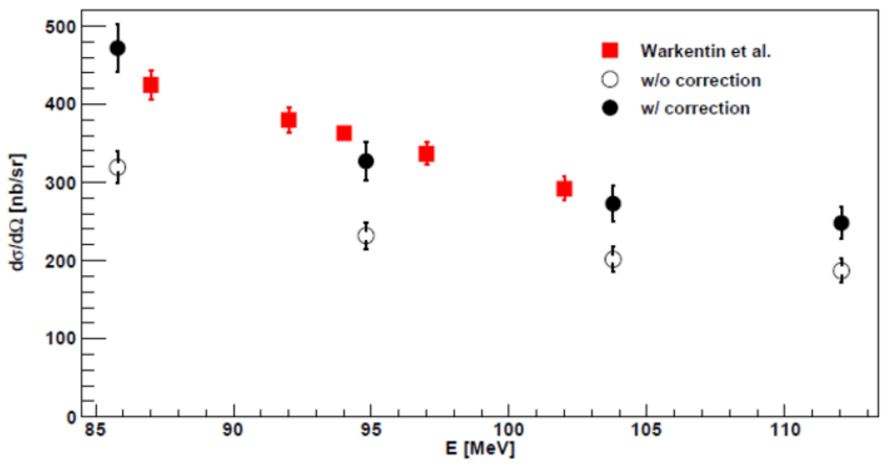

Figure 3. The scattering cross section for ${ }^{12} C$ is shown as a function of photon energy at $\theta_{\gamma}$ $=60^{\circ}$. The read squares are data obtained by [4]. Circles are from the experiment at the MAX IV Laboratory with and without corrections for stolen trues.

\subsection{Measurements of the Giant Resonance in ${ }^{4} \mathrm{He}$}

The goal of this experiment is to measure the total photoabsorption cross section $\sigma\left(\mathrm{E}_{\gamma}\right)$ of ${ }^{4} \mathrm{He}$ in the energy range from 15 to $65 \mathrm{MeV}$. Recent $\mathrm{Ab}$ initio [5] calculations may then be compared to experimental data. A ${ }^{4} \mathrm{He}$ active target with a pressure of $2 \mathrm{MPa}$ was used, see Fig. 4 for its characteristics. To the ${ }^{4} \mathrm{He}$ gas an admixture of $1000 \mathrm{ppm} \mathrm{N}_{2}$ was added in order to shift the VUV light to the spectral range $\sim 420 \mathrm{~nm}$. The target may also be used in investigations of other reaction, e.g. $(\gamma, \gamma)$ and $(\gamma, \mathrm{n})$ in ${ }^{4} \mathrm{He}$ and ${ }^{3} \mathrm{He}$.

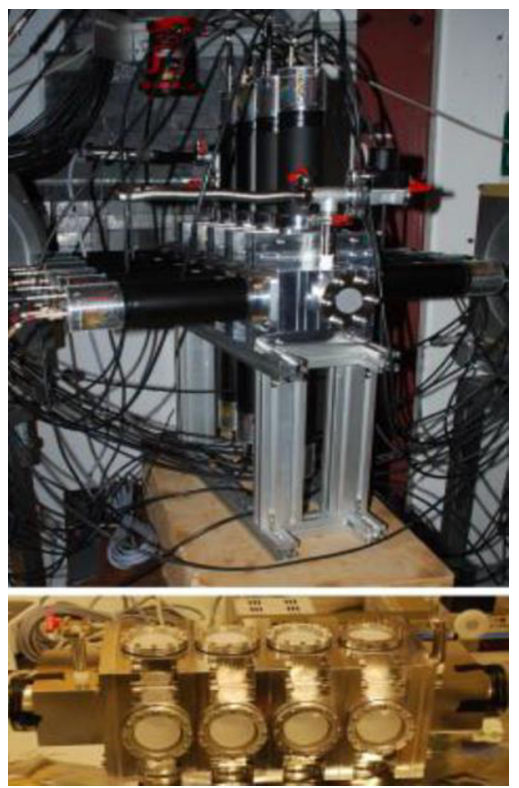

Figure 4. The active ${ }^{4} \mathrm{He}$ target consists of 4 separate cells. Each cell is viewed by four PM tubes where the shifted scintillation light is registered. The lower part of the figure shows the active target before the PM tubes were attached. The target was designed and constructed at the University of Glasgow [6] and used at the MAX IV facility in the PhD project of R. Al Jebali. The upper part of the figure shows the gas cell placed in the photon beam with the PM tubes connected. The target assembly consists, apart from the main cells, of two isolation cells placed at either end of the target with a single PM tube attached to each cell. These outer cells isolate the main cells from particles produced in the pressure holding windows. Each of the main cells is $72 \mathrm{~mm}$ wide and the total target thickness of the active target is about $100 \mathrm{mg} / \mathrm{cm}^{2}$. 
A pilot experiment was carried out at the MAX IV Laboratory with tagged photons in the energy range from 12.7 to $60.5 \mathrm{MeV}$. The average $\Delta \mathrm{E}_{\gamma}$ was $\sim 0.8 \mathrm{MeV}$. In this experiment corrections for stolen trues were again an issue, thus the measurements will continue with different rates of tagged photons. It was nevertheless possible to extract preliminary values for the $\sigma\left(\mathrm{E}_{\gamma}\right)$ of ${ }^{4} \mathrm{He}$. The data is compared to cloud chamber measurements by Arkatov et al. [7]. Theoretical calculations [5, 8] predict the peak of the Giant Resonance of ${ }^{4} \mathrm{He}$ to be $3.4 \mathrm{mb}$ at $27 \mathrm{MeV}$, in good agreement with the data in Fig. 5.

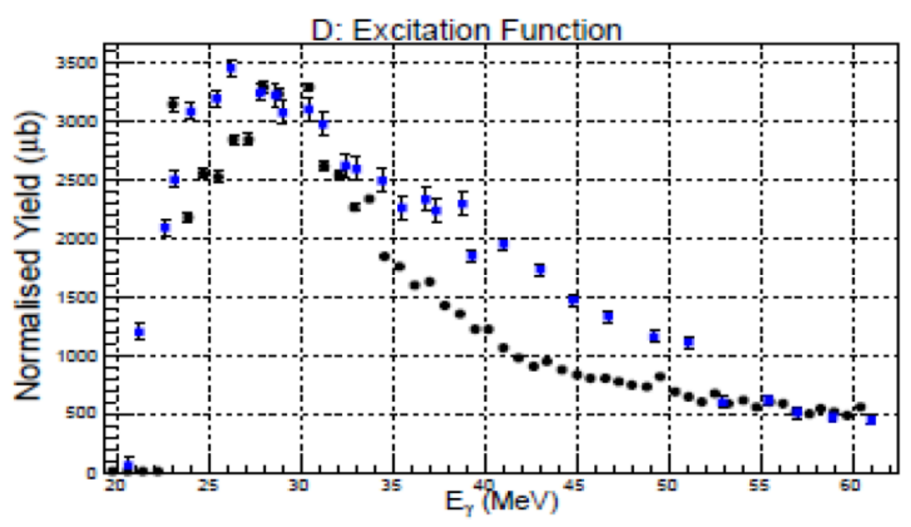

Figure 5. The total photoabsorption cross section for ${ }^{4} \mathrm{He}$ is shown as a function of photon energy (black circles). The data are compared to measurements by Arkatov et al. [7] (blue squares). The MAX IV Laboratory data are PRELIMINARY.

\section{Summary}

The tagged photon facility at the MAX IV Laboratory was upgraded in 2004 to higher photon energies and an extended experimental area. The facility is used by an international collaboration exploring various photonuclear reactions, e.g. Compton scattering on deuterium and light nuclei (University of Illinois at Urbana-Champaign, The George Washington University, Washington DC, University of Kentucky, Lexington and Duke University, Durham, Lund University, MAX IV Laboratory and University of Glasgow), $\left(\gamma, \pi^{+}\right)$reactions in $\mathrm{H},{ }^{2} \mathrm{H},{ }^{6} \mathrm{Li},{ }^{12} \mathrm{C}$ and ${ }^{27} \mathrm{Al}$ (Lund University, MAX IV Laboratory, The George Washington University, University of Massachusetts Dartmouth), and measurements of the total photoabsorption cross section of ${ }^{4} \mathrm{He}$ and ${ }^{6,7} \mathrm{Li}$ (University of Glasgow and University of Tübingen).

\section{References}

[1] J.-O. Adler et al., Nucl. Instr. and Meth. A715, 1 (2013)

[2] L. Myers et al., Nucl. Instr. and Meth. A729, 707 (2013)

[3] H.W. Griesshammer, Prog. Part. Nucl. Phys. 67, 841 (2012)

[4] B.J. Warkentin et a., Phys. Rev. C64, 014603 (2001)

[5] W. Leidemann and G. Orlandini, arXiv:1204.4617v2

[6] S. Al Jebali and J.R.M. Annand, private communication

[7] Y.M. Arkatov et al., Sov. J. Nucl. Phys. 19, 598 (1974)

[8] W. Horiuchi et al., Phys. Rev. C85, 054002 (2012) 